\title{
Detectability of gamma-ray emission from classical novae with Swift/BAT
}

\author{
F. Senziani ${ }^{1,2,3,4}$, G. K. Skinner ${ }^{5,6}$, P. Jean ${ }^{4}$, and M. Hernanz ${ }^{7}$ \\ 1 Università di Pavia, Dipartimento di Fisica Nucleare e Teorica, via Agostino Bassi 6, 27100 Pavia, Italy \\ 2 Istituto Universitario di Studi Superiori (IUSS), V. le Lungo Ticino Sforza 56, 27100 Pavia, Italy \\ INAF - Istituto di Astrofisica Spaziale e Fisica Cosmica, via Bassini 15, 20133 Milano, Italy \\ e-mail: senziani@iasf-milano.inaf.it \\ 4 CESR/Université Toulouse III, 9 avenue du Colonel Roche, 31028 Toulouse, France \\ 5 NASA/Goddard Space Flight Center, Code 661, Greenbelt, MD 20771, USA \\ 6 CRESST/Dept. of Astronomy, University of Maryland CP, College Park, MD 20742, USA \\ 7 IEEC-CSIC, Campus UAB, Fac. Cienc., C5-par, 2on, 08193 Bellaterra, Spain
}

Received 27 March 2008 / Accepted 21 April 2008

\section{ABSTRACT}

\begin{abstract}
Context. Classical novae are expected to emit gamma rays during their explosions. The most important contribution to the early gamma-ray emission comes from the annihilation with electrons of the positrons generated by the decay of ${ }^{13} \mathrm{~N}$ and ${ }^{18} \mathrm{~F}$. The photons are expected to be down-scattered to a few tens of $\mathrm{keV}$, and the emission is predicted to occur some days before the visual discovery and to last $\sim 2$ days. Despite a number of attempts, no positive detections of such emission have been made, due to lack of sensitivity and of sky coverage.

Aims. Because of its huge field of view, good sensitivity, and well-adapted (14-200 keV) energy band, Swift/BAT offers a new opportunity for such searches. BAT data can be retrospectively used to search for prompt gamma-ray emission from the direction of novae after their optical discovery.

Methods. We have estimated the expected success rate for the detection with BAT of gamma rays from classical novae using a Monte Carlo approach. Searches were performed for emission from novae occurring since the launch of Swift.

Results. Using the actual observing programme during the first 2.3 years of BAT operations as an example, and sensitivity achieved, we estimate the expected rate of detection of classical novae with BAT as $\sim 0.2-0.5 \mathrm{yr}^{-1}$, implying that several should be seen within a $10 \mathrm{yr}$ mission. The search for emission in the directions of the 24 classical novae discovered since the Swift launch yielded no positive results, but none of these was known to be close enough for this to be a surprise. Detections of a recurrent nova (RS Oph) and a nearby dwarf nova (V455 And) demonstrate the efficacy of the technique.

Conclusions. The absence of detections is consistent with the expectations from the Monte Carlo simulations, but the long-term prospects are encouraging given an anticipated Swift operating lifetime of $\sim 10$ years.
\end{abstract}

Key words. gamma rays: observations - nuclear reactions, nucleosynthesis, abundances - stars: novae, cataclysmic variables stars: white dwarfs

\section{Introduction}

Classical novae are thought to be cataclysmic variables in which a white dwarf (WD) accretes material from a companion star until the conditions for hydrogen ignition are reached on its surface and a thermonuclear runaway sets in, leading to an explosion (Starrfield et al. 1978). As first noted by Clayton \& Hoyle (1974), novae are expected to emit gamma rays associated with the decay of the radioactive isotopes produced during the explosion. It is largely the decay of the short-lived $\beta^{+}$-unstable nuclei, such as ${ }^{13} \mathrm{~N},{ }^{14,15} \mathrm{O}$ and ${ }^{17,18} \mathrm{~F}$, that leads to a sudden release of a great amount of energy, and the consequent envelope expansion, huge luminosity increase and mass ejection. The positrons emitted in the decay annihilate with electrons, producing a characteristic $511 \mathrm{keV}$ line. Comptonization of most of the $511 \mathrm{keV}$ photons also leads to a continuum down to a few tens of $\mathrm{keV}$. Among the short-lived isotopes, ${ }^{13} \mathrm{~N}$ and ${ }^{18} \mathrm{~F}$ (with lifetime $\tau=$ $862 \mathrm{~s}$ and 158 min respectively) are expected to be the most important contributors to the prompt gamma-ray emission since they decay when the envelope is starting to become transparent.
Later gamma-ray emission comes from the decay of mediumand long-lived isotopes, such as ${ }^{7} \mathrm{Be}$ (lifetime $\tau=77$ days), ${ }^{22} \mathrm{Na}$ ( $\tau=3.75 \mathrm{yrs}$ ), and ${ }^{26} \mathrm{Al}$ ( $\left.\tau=10^{6} \mathrm{yrs}\right)$. Photons are emitted by these nuclei with characteristic energies of $478 \mathrm{keV}$ for ${ }^{7} \mathrm{Be}$, $1275 \mathrm{keV}$ for ${ }^{22} \mathrm{Na}$ and $1809 \mathrm{keV}$ for ${ }^{26} \mathrm{Al}$. Because of their longer decay times, the decay rates of these isotopes is much lower than that of ${ }^{13} \mathrm{~N}$ or ${ }^{18} \mathrm{~F}$ and they are not considered here.

The gamma-ray emission depends critically on the nature and characteristics of the thermonuclear explosion, so its observation would provide a sensitive test of our theories of classical novae. However, to date no detection has been possible. Because novae are unpredictable, detecting prompt gamma rays from novae requires a wide field of view (FOV) telescope that can continuously monitor the sky with good sensitivity over the appropriate energy range. Unsuccessful attempts have been made using BATSE on board CGRO (Hernanz et al. 2000), TGRS on board WIND (Harris et al. 1999, 2000), and RHESSI (Smith 2004). Hernanz \& José (2004) presented prospects for the detectability of the electron-positron annihilation continuum with IBIS/ISGRI telescope on board INTEGRAL. Despite the good 
sensitivity of the instrument, they found that a detection is expected only if a nova closer than $4-5 \mathrm{kpc}$ lies by chance in the IBIS FOV within about $10 \mathrm{~h}$ of the outburst temperature peak. The relatively small FOV $\left(29^{\circ} \times 29^{\circ}\right)$ of the IBIS telescope makes the probability of this very low and indeed, it has not occurred during the five years since the launch of INTEGRAL.

The Burst Alert Telescope (BAT) on board the Swift satellite (Gehrels et al. 2004) offers a new opportunity to search for the prompt gamma rays from novae. The BAT satisfies all the requirements described above: it has a FOV of $\sim 2$ sr (about an order of magnitude larger than that of IBIS) and covers $\sim 50 \%$ of the sky each day. It has a sensitivity similar to that of IBIS/ISGRI and works in an energy range (14-200 keV) that is well matched to that of the expected nova emission.

In this paper we investigate the detectability of the prompt gamma-ray emission from classical novae with the BAT. We first discuss the expected fluxes in the BAT energy range based on the latest available nova models for the production of ${ }^{13} \mathrm{~N}$ and ${ }^{18} \mathrm{~F}$ (Sect. 2). A description of the BAT performance and of the approach we adopted to search for prompt gamma rays from novae is given in Sect. 3. In Sect. 4 we use a Monte Carlo approach to investigate the probability of detecting the expected emission based on modelling the distribution of novae in the Galaxy and on the characteristics of the Swift observing sequence. Results of a retrospective search in the BAT data archive for gamma rays from 24 novae discovered during the first 3 years of Swift operations are presented in Sect. 5.

\section{The expected prompt gamma-ray emission from novae}

The gamma-ray flux expected during the explosion of a typical classical nova depends on the amount of the isotopes ${ }^{13} \mathrm{~N}$ and ${ }^{18} \mathrm{~F}$ produced, and on the probability that the gamma rays resulting from their decay can escape. This flux has been computed with a suite of two numerical codes: the hydrodynamical code developed to simulate a nova explosion and its nucleosynthesis, from the accretion up to the explosion stages (José \& Hernanz 1998), and the Monte Carlo code dealing with the production and transfer of gamma rays in the expanding nova envelope (Gómez-Gomar et al. 1998). The results depend on the mass of the WD and its chemical composition ( $\mathrm{CO}$ or $\mathrm{ONe})$, the initial luminosity, the degree of mixing between the WD core and the accreted envelope and the mass accretion rate; typical masses adopted in the simulations are $1.25 M_{\odot}$ for ONe-type and $1.15 M_{\odot}$ for CO-type novae, with degrees of mixing of $50 \%$, accretion rate $2 \times 10^{-10} M_{\odot} \mathrm{yr}^{-1}$ and $L_{\text {in }}=1 \times 10^{-2} L_{\odot}$ (see Hernanz et al. 2002). The results also depend critically on nuclear cross-sections, some of which are poorly known (see Hernanz \& José 2006). Important reductions in the ${ }^{18} \mathrm{~F}$ yields with respect to models used in Gómez-Gomar et al. (1998) have been reported in Hernanz et al. (1999) and Coc et al. (2000); the nova models from these papers have been used here, and the expected reduction in the gamma-ray flux due to the new ${ }^{18} \mathrm{~F}$ yields obtained with the recent Chafa et al. (2005) cross sections has been applied as a correcting factor in the second peak of the light curves (see below). The successive reductions in the ${ }^{18} \mathrm{~F}$ yield along the past 10 years is as follows, if we adopt the yields with the rates of Chafa et al. (2005) as a reference: a reduction by a factor of $\sim 8$ with respect to Coc et al. (2000), a factor of $\sim 50$ with respect to Hernanz et al. (1999), and a factor of 500 with respect to Gómez-Gomar et al. (1998).

The situation concerning the more recent determinations of nuclear cross-section affecting ${ }^{18} \mathrm{~F}$ synthesis is not completely
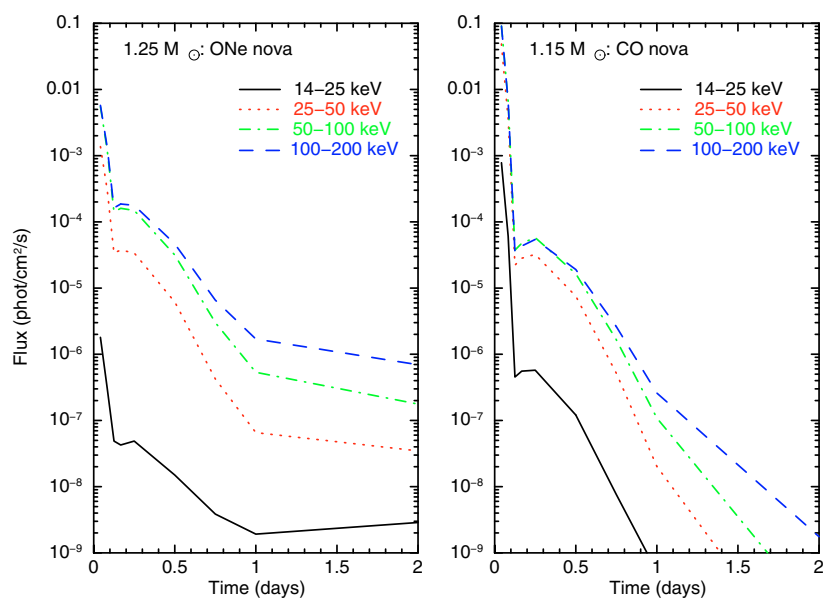

Fig. 1. Lightcurves in four energy bands for a $1.25 M_{\odot}$ ONe-type nova (left) and for a $1.15 M_{\odot}$ CO-type nova (right) based on the most recent available nova models and for a distance of $1 \mathrm{kpc}$.

settled yet, but at least the uncertainties are becoming much smaller.

To assess the detectability with BAT of prompt gamma-ray emission from novae, we ran the two codes mentioned for the above conditions of mass and other parameters corresponding to a $\mathrm{CO}$ and an ONe nova. The resulting predicted lightcurves in four energy bands are shown in Fig. 1. The strongest peak is due to the decay of ${ }^{13} \mathrm{~N}$ and reaches a maximum at $t \sim 1 \mathrm{~h}$, the flux at earlier times being reduced by the envelope opacity. The second peak at $t \sim 6 \mathrm{~h}$ is from the decay of ${ }^{18} \mathrm{~F}$. Depending on the nova speed class (Gómez-Gomar et al. 1998), the peak gamma-ray emission is expected to occur some days before the visual maximum and hence probably before the discovery. Thus it is most likely to be detected if retrospective searches are made in the data from an instrument with good sky coverage. The ${ }^{18} \mathrm{~F}$ emission is longer lasting and so, with an instrument making many different pointings, is easier to detect, provided the sensitivity is good enough. However the ${ }^{13} \mathrm{~N}$ peak is stronger and so detectable at greater distance.

\section{The Swift/BAT instrument}

As discussed in Sect. 1, the BAT (Barthelmy et al. 2005) on board Swift is well adapted to search for prompt gamma rays from novae. It is a coded-mask telescope operating in the $15-150 \mathrm{keV}$ energy range. The BAT detector plane is composed of 32768 pieces of CdZnTe $(\mathrm{CZT})$, covering a $1.2 \times 0.6 \mathrm{~m}$ sensitive area. A $2.7 \mathrm{~m}^{2} \mathrm{D}$-shaped coded mask, with $\sim 54000$ lead tiles arranged in a random, $50 \%$ open pattern, is mounted $1 \mathrm{~m}$ above the detector plane. The instrument FOV covers $\sim 1 / 6$ of the sky in a single pointing. Within the field of view the sensitivity depends on the "coding fraction" which varies from 1 ("fully coded") in the central $0.5 \mathrm{sr}$ of the field, to 0 at its limits. The FOV with a coded fraction greater than 0.1 is about $2.2 \mathrm{sr}(1.5 \mathrm{sr}$ with coding $>0.5$ ).

For exposures of duration $\Delta t$, the $5 \sigma$ sensitivity $\left(\mathrm{ph} / \mathrm{cm}^{2} / \mathrm{s}\right)$ for a point source at a coded fraction of $n_{\text {c.f. }}$ is given by

$$
S(E)=\frac{5 \cdot \sqrt{r_{\mathrm{B}}(E)}}{\left(A_{\mathrm{det}} / 2\right) \cdot \eta(E) \cdot f_{\mathrm{m}} \cdot \sqrt{n_{\text {c.f. }} \cdot \Delta t}}
$$

where $r_{\mathrm{B}}(E)$ and $\eta(E)$ are the background count rate in the whole detector and the detector efficiency in the energy band under consideration. $A_{\text {det }}$ is the detector plane area and $f_{\mathrm{m}}$ (equal to 0.73 
Table 1. Models of the nova spatial distribution in the Galaxy used for the Monte Carlo simulations (Jean et al. 2000).

\begin{tabular}{|c|c|c|}
\hline \multicolumn{3}{|c|}{$\begin{array}{l}\text { Model } 1 \text { (Kent et al. 1991): } \\
\rho_{\mathrm{h}}=3.0 \mathrm{kpc} \text { and } z_{\mathrm{h}}=0.170 \mathrm{kpc} . K_{0} \text { is the modified Bessel function }\end{array}$} \\
\hline \multirow{4}{*}{$\begin{array}{l}\text { Disc } n(z, \rho) \\
\text { Bulge } n(R)\end{array}$} & $=n_{\mathrm{d}} \exp \left(-\frac{|\mathrm{z}|}{z_{\mathrm{h}}}-\frac{\rho}{\rho_{\mathrm{h}}}\right)$ & \\
\hline & $=n_{\mathrm{s}} 1.04 \times 10^{6}\left(\frac{R}{0.482}\right)^{-1.85}$ & $R \leq 0.938 \mathrm{kpc}$ \\
\hline & $=n_{\mathrm{s}} 3.53 K_{0}\left(\frac{R}{0.667}\right)$ & $R \geq 0.938$ \\
\hline & $=0$ & $R \geq 5 \mathrm{kpc}$ \\
\hline \multicolumn{3}{|c|}{$\begin{array}{l}\text { Model } 2 \text { (King et al. 1990): } \\
\rho_{\mathrm{h}}=5.0 \mathrm{kpc} \text { and } z_{\mathrm{h}}=0.30 \mathrm{kpc}\end{array}$} \\
\hline \multirow{3}{*}{$\begin{array}{l}\text { Disc } n(z, \rho) \\
\text { Bulge } n(R)\end{array}$} & $=n_{\mathrm{d}} \exp \left(-\frac{|z|}{z_{\mathrm{h}}}-\frac{\rho}{\rho_{\mathrm{h}}}\right)$ & \\
\hline & $=n_{\mathrm{s}} 1.25\left(\frac{R}{R_{\mathrm{g}}}\right)^{-6 / 8} \exp \left[-10.093\left(\frac{R}{R_{\mathrm{g}}}\right)^{1 / 4}+10.093\right]$ & $R \leq R_{\mathrm{g}}$ \\
\hline & $=n_{\mathrm{s}}\left(\frac{R}{R_{\mathrm{g}}}\right)^{-7 / 8}\left[1-\frac{0.0867}{\left(\frac{R}{R_{\mathrm{g}}}\right)^{1 / 4}}\right] \exp \left[-10.093\left(\frac{R}{R_{\mathrm{g}}}\right)^{1 / 4}+10.093\right]$ & $R \geq R_{\mathrm{g}}$ \\
\hline \multicolumn{3}{|c|}{$\begin{array}{l}\text { Model } 3 \text { (Dawson \& Johnson 1994): } \\
\rho_{\mathrm{h}}=5.0 \mathrm{kpc} \text { and } z_{\mathrm{h}}=0.35 \mathrm{kpc} \\
\end{array}$} \\
\hline Disc $n(z, \rho)$ & $=n_{\mathrm{d}} \exp \left(-\frac{|z|}{z_{\mathrm{h}}}-\frac{\rho}{\rho_{\mathrm{h}}}\right)$ & \\
\hline Bulge $n(R)$ & $\begin{array}{l}=\frac{n_{\mathrm{s}}}{R^{3}+0.343} \\
=0\end{array}$ & $\begin{array}{l}R \leq 3 \mathrm{kpc} \\
R \geq 3 \mathrm{kpc}\end{array}$ \\
\hline
\end{tabular}

$R$ is the distance from the Galactic Centre, $z$ is the distance perpendicular to the Galactic plane and $\rho$ is the galactocentric planar distance. The distance from the Galactic Centre to the Sun is $R_{\mathrm{g}}=8 \mathrm{kpc}, n_{\mathrm{s}}$ and $n_{\mathrm{d}}$ are the normalisation factors for the bulge and the disc respectively (in kpc ${ }^{-3}$ ). The proportions of novae in the bulge are 0.179 (Model 1), 0.105 (Model 2) and 0.111 (Model 3).

for BAT) is a factor which takes into account the size of the detector pixels relative to the mask elements (Skinner 2008).

The Swift observing strategy has important implications for the search for prompt emission from novae. As the Swift satellite is specifically designed to catch and study gamma-ray bursts (GRBs), the BAT field is generally directed away from the earth. Several different pointings are made in each spacecraft orbit (96 min). The directions are chosen such that its companion narrow-field instrument, the XRT (X-ray Telescope), can observe particular objects or follow-up GRB afterglows. As the XRT targets are well distributed over the sky, the BAT is able to collect "survey mode" data from a large fraction of the sky every day. In survey mode, while awaiting GRBs, data are accumulated on board in arrays, called Detector Plane Histograms (DPHs), that are periodically sent to the ground. A DPH contains a 80-channel spectrum for each of the 32768 pixels of the detector plane. Such histograms are integrated over $450 \mathrm{~s}$, or sometimes less if the integration is terminated for operational reasons such as the start of a spacecraft slew or the approach to the South Atlantic Anomaly. On board software searches BAT event rates and images for increases corresponding to GRBs, working over a range of time scales (see Gehrels et al. 2004; Fenimore et al. 2003). The prompt gamma-ray emission from a nova could in principle trigger the GRB detection system and lead to a spacecraft repointing, but it would probably be too faint and longlasting to be detected in this way. However, once it is known that a nova has occurred at a particular location, a more sensitive retrospective search is possible. Thus the survey mode DPH data provides a valuable resource for nova emission searches.

A particular direction on the sky is typically within the field of view many times per day, sometimes in an irregular pattern, sometimes with consecutive observations spaced by the 96 min orbital period. This sampling pattern is particularly relevant for the detection of novae because their peak flux could be missed during the gaps.

\section{Prospects for detection}

\subsection{Monte Carlo simulations}

Whether or not prompt gamma-ray emission from a particular nova can be detected with BAT depends in a complex way on the distance and gamma-ray luminosity of the nova and how the gamma-ray light curve is sampled by the sequence of pointings for which it lies within the field of view. To estimate the probable rate of detection of novae with BAT, we generated a sample of imaginary novae with a Monte Carlo simulation, that were checked against a typical observing sequence. For the observing programme we used the actual BAT pointings made during the first 2.3 years of operation.

\subsubsection{Nova distribution models}

Novae were simulated based on each of three different models of spatial distribution in the Galaxy. The models were those used by Jean et al. (2000), excluding their oldest population model. Details of the three adopted models are summarised in Table 1. Each has a different distribution between the novae in the bulge and those in the disk.

\subsubsection{The rate of novae}

The rate of occurrence of classical novae in the Galaxy has been variously estimated as $35 \pm 11 \mathrm{yr}^{-1}$ (Shafter 1997), $41 \pm 20 \mathrm{yr}^{-1}$ (Hatano et al. 1997) and 34 $4_{-12}^{+15} \mathrm{yr}^{-1}$ (Darnley et al. 2006).

However, to be the subject of a retrospective search in BAT data, a nova has first to be discovered in the optical domain. Thus the relevant rate is strictly the frequency of reported 

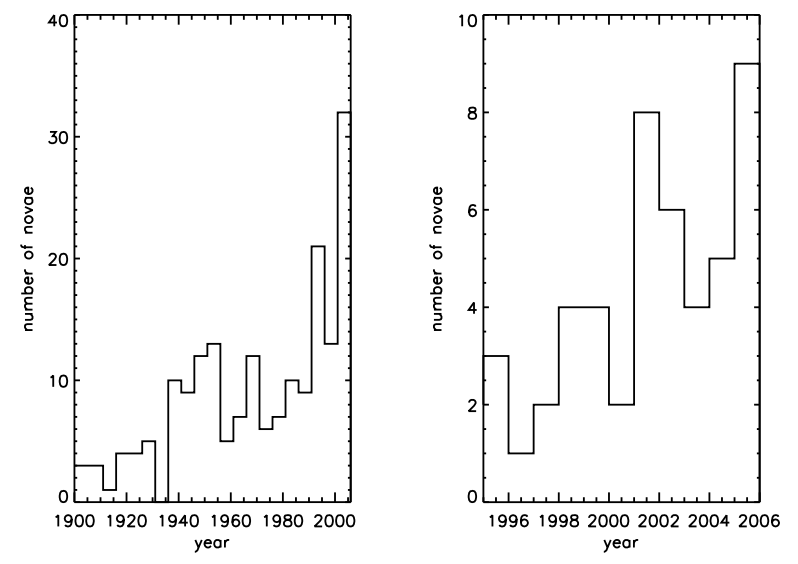

Fig. 2. Histogram of the novae discovered in 5 years intervals from 1901-2005 (left) and per year from 1995-2005 (right).

classical novae. Interestingly, the discovery of a classical nova depends mainly on the work of amateurs astronomers. A very useful catalogue is available on the web, containing all novae discovered up to February 1, 2006 (see Downes et al. 2001, and the web-site http://archive.stsci.edu/prepds/cvcat/ index.html). From that, we selected all those objects classified as classical novae. The number discovered in 5 year intervals over the last century is shown in the left panel of Fig. 2.

The general rising trend in the number of novae discovered per year is certainly related to the steadily increasing activities of amateurs. The righthand panel of Fig. 2 shows the number discovered during each of the last ten years. The rate has recently risen to $\left(\sim 10 \mathrm{yr}^{-1}\right)$, but is still far from the predicted Galactic nova rate. This is mainly due to interstellar extinction, though at any one time a part of the sky is too close to the sun for a nova to be observed.

\subsubsection{White dwarf composition}

According to the calculations described in Sect. 2, at very early times CO novae release more gamma rays related to ${ }^{13} \mathrm{~N}$ decay than $\mathrm{ONe}$ ones, so for the simulations we make an assumption about the relative numbers of $\mathrm{ONe}$ and $\mathrm{CO}$ novae. We note however that this difference is probably not a general trend, since it is strongly related to the efficiency of convection in the outer nova layers, and thus a range of fluxes between those presented here is expected, making the results less dependent on the assumption made.

The classification of a nova as type $\mathrm{ONe}$ or $\mathrm{CO}$ relies on spectroscopic observations and is not always available. Livio \& Truran (1994) estimated that ONe novae represent between $11 \%$ and $33 \%$ of galactic novae. The results presented here are based on a mix of $25 \% \mathrm{ONe}$ and $75 \% \mathrm{CO}$ novae, both in the disc and in the bulge, but results are also given for $\mathrm{ONe}$ and for $\mathrm{CO}$ novae separately.

We note that the expected gamma-ray flux from a nova depends mainly on the WD mass (see Hernanz et al. 2002) and the fluxes shown in Fig. 1 were calculated assuming single, typical, WD masses for ONe and for CO novae. In this respect, the approach adopted here is a somewhat simplified one.

\subsection{Monte Carlo results}

For each distribution model, the positions in the Galaxy and the time of explosion of 350000 imaginary novae were generated,

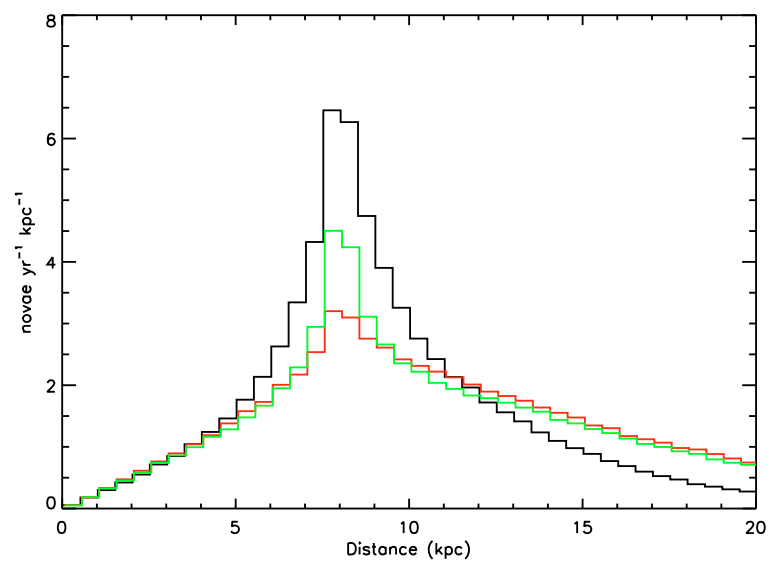

Fig. 3. Distribution of the distances from the Sun of the simulated novae. Black, red and green histograms correspond to novae distributed according to models $1-3$ respectively (see Table 1 ).

finding for each one a distance from the Sun and the sky coordinates. All of the explosion times chosen were during the first 2.3 years of BAT observations, for which we know the actual observing sequence and observing efficiency. Each nova was allocated to a class $(\mathrm{ONe}$ or $\mathrm{CO})$. The distributions of the distances from the Sun of the simulated novae is shown in Fig. 3 for each of the three models.

We then assessed whether BAT would have seen the gamma rays from the novae. If and when during the $48 \mathrm{~h}$ following the nova it would have been within the BAT field of view, the sensitivity for a source at the corresponding position in the FOV was calculated using Eq. (1). A nova was assumed to have been detected if the expected flux from Fig. 1, integrated over the observation time and scaled to the distance of the simulated nova, exceeded the $5 \sigma$ sensitivity.

Figure 4 shows the distribution of the distances of the detectable novae according to the Monte Carlo simulation. Although close novae are more easily detected, more distant ones are more numerous, leading to a peak at $\sim 2-3 \mathrm{kpc}$, although detection of novae at distances up to $\sim 5 \mathrm{kpc}$ is possible in favourable circumstances.

These results allow one to calculate the probability that, if a nova explodes somewhere in the Galaxy, the gamma rays would be detectable with the BAT. This can be multiplied by an appropriate nova rate (Sect. 4.1.2) to give the expected detection rate.

The results for model 1 of Table 1 are given in Table 2 . We have not given explicitly the results for the other two models because they are essentially identical. In calculating the detection probability, it is considered that a nova would be detected if it would have been seen at a significance level greater than $5 \sigma$ using any of the following integration timescales: single DPH ( $13 \mathrm{~min}), 1 \mathrm{~h}, 3 \mathrm{~h}, 6 \mathrm{~h}, 12 \mathrm{~h}$ and $24 \mathrm{~h}$. Usually it is the single DPH or $1 \mathrm{~h}$ timescales that prove most sensitive.

Table 2 shows that the $50-100 \mathrm{keV}$ band is the most promising one and that the probability of detection of a particular nova is only about $1.5 \%$. Nevertheless, with a nova rate of $35 \mathrm{yr}^{-1}$ and a hoped-for mission life of $10 \mathrm{yr}$, this implies an expectation number of 5.2 detected novae. However, as discussed in Sect. 4.1.2, a nova must be discovered optically in order to perform the retrospective search for gamma-ray emission. At first sight this would imply that an effective rate of $\sim 10 \mathrm{yr}^{-1}$ should be multiplied by $1.5 \%$, suggesting only 1.5 over a $10 \mathrm{yr}$ Swift mission. 


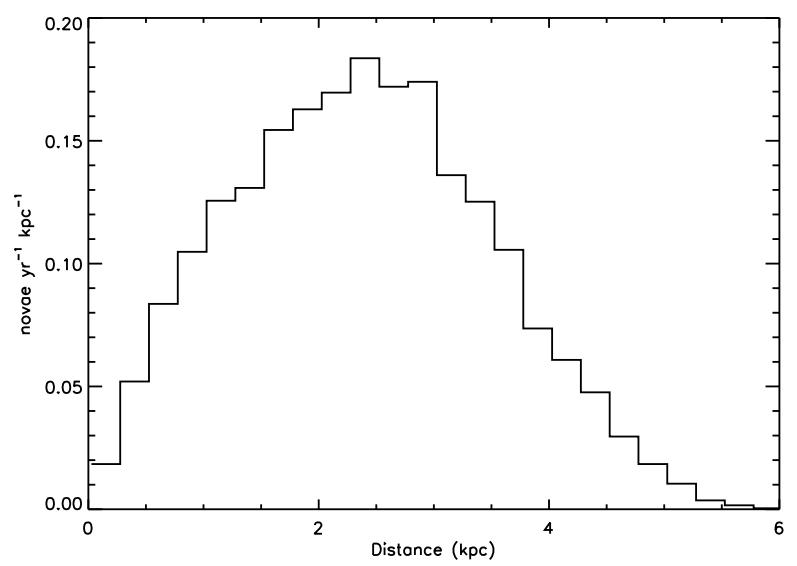

Fig. 4. Distribution of the distances from us of the novae detected in gamma rays in the Monte Carlo simulation. Model 1 was assumed.

Table 2. The probability that a novae is detectable with BAT, for each of four energy bins. Results are for the spatial distribution of model 1 in Table 1. Other distribution models give very similar results (see text for details).

\begin{tabular}{ccccc}
\hline \hline $\begin{array}{c}\text { Nova type } \\
\text { proportions }\end{array}$ & \multicolumn{5}{c}{ Detection probabilities } \\
\hline & $14-25 \mathrm{keV}$ & $25-50 \mathrm{keV}$ & $50-100 \mathrm{keV}$ & $100-200 \mathrm{keV}$ \\
\hline $100 \%$ ONe & 0 & $0.02 \%$ & $0.12 \%$ & $0.10 \%$ \\
$100 \% \mathrm{CO}$ & $0.007 \%$ & $0.82 \%$ & $1.95 \%$ & $1.59 \%$ \\
$25 \% \mathrm{ONe}+75 \% \mathrm{CO}$ & $0.004 \%$ & $0.63 \%$ & $1.55 \%$ & $1.25 \%$ \\
\hline
\end{tabular}

However the novae that are discovered optically are preferentially those that are close, both because they are brighter and because interstellar absorption tends to be less. We have examined the distribution in $m_{V}$ of the discovered novae in Downes et al. (2001) catalog, considering novae discovered between 1985 and 2005. This can be compared with the corresponding distribution that would be expected based on our simulated nova population, taking the absolute visual magnitude of a typical nova as -8 and allowing for galactic extinction based on Hakkila et al. (1997). We conclude that although the above rates imply that only about 1 nova in 3-4 is observed, the probability of optical discovery of a nova of apparent magnitude $m_{V}<9$ is about $50 \%$, roughly independent of magnitude. For $m_{V}>9$ it decreases rapidly and is essentially zero for $m_{V}>14$. We used such a probability distribution to weight the distribution in magnitude of the novae detected with BAT among those of our simulated population. Both the weighted and the unweighted distributions of the novae detected with BAT are plotted in Fig. 5.

We conclude that based on the assumptions made here $2-5$ novae should be detectable in gamma rays during a $10 \mathrm{yr}$ mission.

\section{Observational results}

\subsection{Classical novae since the launch of Swift}

The classical novae discovered during the first $\sim 3$ years after the Swift launch are listed in Table 3. Of these, 7 novae have been identified as CO type. Distance measures are available in the literature for only 5 of the novae. Where sufficient data are available we have attempted to make our own distance estimates using the peak magnitude, $m_{V}$, the time to decay by $2 \mathrm{mag}, t_{2}$, the reddening $E(B-V)$ and the MMRD relationship of Della Valle $\&$ Livio (1995). These are given in Table 4. $m_{V}$, and $t_{2}$, were

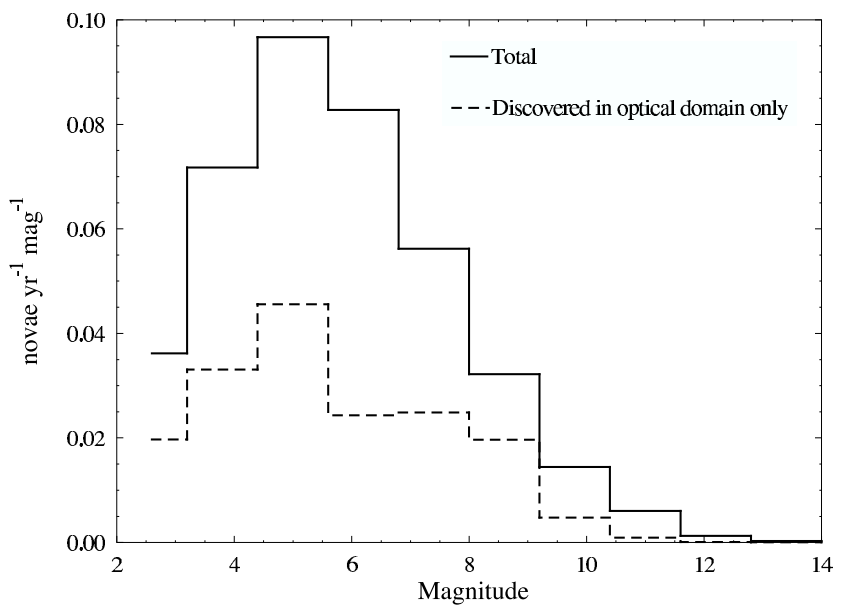

Fig. 5. Distribution of the magnitude of the novae detected in gamma rays in the Monte Carlo simulation (solid line). The dashed line represents the same distribution weighted for the probability of optical discovery of a nova (see text for details).

estimated from AAVSO lightcurves (see http: //www . aavso. org/). $E(B-V)$ values were taken from IAU Circulars when available, otherwise the extinction map of Hakkila et al. (1997) was used (see Table 4). The derived distances must be treated with some caution because of the large uncertainty in the values used and in the MMRD relationship adopted.

\subsection{Analysis}

For each nova, we selected all the BAT observations having the position of the nova in the FOV from 20 days before to 20 days after the nova discovery. The relatively long time interval takes into account the uncertainty in the time difference between the expected prompt gamma-ray emission and the nova discovery (see Sect. 2) and also provides a baseline for comparison.

The DPH files (see Sect. 3 for details) for the selected datasets were analysed with a procedure combining public tools ${ }^{1}$ and a special purpose fitting programme. The data were filtered to exclude periods of high background rates, source occultation, etc. Typically, a DPH file contains information for several consecutive pointings, each lasting $450 \mathrm{~s}$ or less, with an average total exposure time of $\sim 13 \mathrm{~min}$. As the peak flux is expected to occur on a timescale of $\sim 1 \mathrm{~h}$ (see Fig. 1), all of the pointings within a single DPH were combined.

The data were analysed in four energy bands, 14-25, 25-50, 50-100, and 100-200 keV, using a procedure that fits simultaneously a series of background models, the intensities of known strong sources and that of a source at the position of the nova.

For each nova and energy bin, the flux estimates were used to form a lightcurve. All the fluxes measured with a signal to noise ratio (SNR) greater than 4 in one energy band or having SNR greater than 2.5 in at least two energy bands were selected for further analysis. For each DPH in which such high points were found, an image of the FOV was generated using the batffimage public tool and searched (with batcelldetect) for the evidence of the nova, setting a threshold of $5 \sigma$ level. The requirement for detection of a signal in both the lightcurve and the image improves the robustness of any detection.

\footnotetext{
1 Version 2.5 of the Swift software (see http://swift.gsfc.nasa. gov/docs/software/lheasoft) was used.
} 
Table 3. The 24 classical novae examined in the search for the prompt gamma-ray emission.

\begin{tabular}{|c|c|c|c|c|c|c|c|}
\hline Nova & $\begin{array}{l}\mathrm{RA} \\
(\mathrm{deg})\end{array}$ & $\begin{array}{l}\text { Dec } \\
(\operatorname{deg})\end{array}$ & $\begin{array}{l}\text { Date of discovery } \\
\text { (UTC) }\end{array}$ & $\begin{array}{l}\text { Last prediscovery } \\
\text { (UTC) }\end{array}$ & Nova type & $\begin{array}{l}\text { Distance from } \\
\text { literature }(\mathrm{kpc})\end{array}$ & References* \\
\hline V2361 Cyg & 302.329 & 39.814 & 2005 Feb. 10.850 & 2005 Feb. 6 & $\mathrm{CO}, \mathrm{FeII}$ & & $1,2,3,4,5,6,7$ \\
\hline V382 Nor & 244.936 & -51.581 & 2005 Mar. 13.309 & $?$ & FeII & & 8,9 \\
\hline V378 Ser & 267.3523 & -12.999 & 2005 Mar. 18.345 & $?$ & $\mathrm{CO}, \mathrm{FeII}$ & & $10,11,12,13,6$ \\
\hline V5115 Sgr & 274.246 & -25.944 & 2005 Mar. 28.779 & 2005 Mar. 27.464 & & & 14,15 \\
\hline V1663 Aql & 286.302 & 5.236 & 2005 Jun. 9.240 & $?$ & FeII & $\begin{array}{c}2.9 \pm 0.4 \\
5.5 \pm 1 \\
7.3-11.3\end{array}$ & $\begin{array}{l}\text { Boyd \& Poyner (2006) } \\
\text { Lane et al. (2006) } \\
\text { Poggiani }(2006) \\
16,17,18\end{array}$ \\
\hline V5116 Sgr & 274.461 & -30.442 & 2005 Jul. 4.049 & 2005 Jun. 12 & & & $19,20,21,22$ \\
\hline V1188 Sco & 266.089 & -34.276 & 2005 Jul. 25.284 & 2005 Jul. 20 & FeII(?) & & $23,24,25,26$ \\
\hline V1047 Cen & 200.207 & -62.630 & 2005 Sep. 1.031 & 2005 Aug. 12.050 & & & 22 \\
\hline V476 Sct & 278.020 & -6.726 & 2005 Sep. 28.088 & 2005 Sep. 24.629 & FeII & $4 \pm 1$ & $\begin{array}{l}\text { Munari et al. (2006a) } \\
27,28,29\end{array}$ \\
\hline V477 Sct & 279.678 & -12.271 & 2005 Oct. 11.026 & 2005 Oct. 7.055 & $\mathrm{He} / \mathrm{N}$ & $11 \pm 3.6$ & $\begin{array}{l}\text { Munari et al. (2006b) } \\
30,31\end{array}$ \\
\hline V2575 Oph & 263.304 & -24.351 & 2006 Feb. 8.379 & 1991 Aug. 11 & $\mathrm{CO}, \mathrm{FeII}$ & & $32,33,34,35$ \\
\hline V5117 Sgr & 269.719 & -36.793 & 2006 Feb. 17.370 & 2006 Feb. 5.36 & FeII & & 36,37 \\
\hline V2362 Cyg & 317.885 & 44.801 & 2006 Apr. 2.807 & 2006 Mar. 28 & $\mathrm{CO}, \mathrm{FeII}$ & $\begin{array}{l}\sim 1.5 \\
5-12\end{array}$ & $\begin{array}{l}\text { Czart et al. (2006) } \\
\text { Steeghs et al. (2006) } \\
38,39,40,34,41,42,43\end{array}$ \\
\hline V2576 Oph & 258.887 & -29.161 & 2006 Apr. 6.565 & $?$ & $\mathrm{CO}, \mathrm{FeII}$ & & $44,34,45$ \\
\hline V1065 Cen & 175.793 & -58.067 & 2007 Jan. 23.354 & 2007 Jan. 15.36 & & & 46,47 \\
\hline V1280 Sco & 254.420 & -32.343 & 2007 Feb. 4.854 & 2007 Feb. 2.866 & FeII & & $\begin{array}{l}\text { Swank (2007) } \\
\text { Osborne et al. (2007) } \\
48,49,50,61\end{array}$ \\
\hline V1281 Sco & 254.247 & -35.363 & 2007 Feb. 19.859 & 2007 Feb. 14.8575 & & & $51,52,62$ \\
\hline V2467 Cyg & 307.052 & 41.810 & 2007 Mar. 15.787 & 2007 Mar. 12.796 & FeII & $1.5-4$ & $\begin{array}{l}\text { Steeghs et al. (2007) } \\
53,63,64,65\end{array}$ \\
\hline V2615 Oph & 265.683 & -23.676 & 2007 Mar. 19.812 & 2007 Mar. 17.82 & $\mathrm{CO}, \mathrm{FeII}$ & & 54,62 \\
\hline V5558 Sgr & 272.577 & -18.781 & 2007 Apr. 14.777 & 2007 Apr. 9.8 & FeII & & $55,56,66,67$ \\
\hline V598 Pup & 106.428 & -38.245 & 2007 Jun. 5.968 & 2007 Jun. 2.978 & $\mathrm{CO}$ & & $\begin{array}{l}\text { Read et al. (2007a) } \\
\text { Torres et al. (2007) } \\
\text { Read et al. (2007b) } \\
76,77,78\end{array}$ \\
\hline V390 Nor & 248.048 & -45.154 & 2007 Jun. 15.086 & 2007 May 20.1 & FeII & & 57,68 \\
\hline V458 Vul & 298.601 & 20.880 & 2007 Aug. 10.01 & 2007 Aug. 4 & & & $69,70,58,71,72,73$ \\
\hline V597 Pup & 124.075 & -34.257 & 2007 Nov. 14.23 & 2007 Nov. 11.22 & FeII & & $59,60,74,75$ \\
\hline
\end{tabular}

* For each nova references to the first few IAU Circulars are given: (1) \#8483; (2) \#8484; (3) \#8487; (4) \#8511; (5) \#8524; (6) \#8529; (7) \#8641; (8) \#8497; (9) \#8498; (10) \#8505; (11) \#8506; (12) \#8509; (13) \#8527; (14) \#8502; (15) \#8523; (16) \#8540; (17) \#8544; (18) \#8640; (19) \#8559; (20) \#8561; (21) \#8579; (22) \#8596; (23) \#8574; (24) \#8575; (25) \#8576; (26) \#8581; (27) \#8607; (28) \#8612; (29) \#8638; (30) \#8644; (31) \#8617; (32) \#8671; (33) \#8676; (34) \#8710; (35) \#8728; (36) \#8673; (37) \#8706; (38) \#8697; (39) \#8698; (40) \#8702; (41) \#8731; (42) \#8785; (43) \#8788; (44) \#8700; (45) \#8730; (46) \#8800; (47) \#8801; (48) \#8803; (49) \#8807; (50) \#8809; (51) \#8810; (52) \#8812; (53) \#8821; (54) \#8824; (55) \#8832; (56) \#8854; (57) \#8850; (58) \#8863; (59) \#8895; (60) \#8896; (61) \#8845; (62) \#8846; (63) \#8848; (64) \#8888; (65) \#8905; (66) \#8874; (67) \#8884; (68) \#8851; (69) \#8861; (70) \#8862; (71) \#8878; (72) \#8883; (73) \#8904; (74) \#8902; (75) \#8911; (76) \#8898; (77) \#8899; (78) \#8901.

If no such double detection was found, the procedure was repeated on lightcurves rebinned with different timebins $(1 \mathrm{~h}, 3 \mathrm{~h}$, $6 \mathrm{~h}, 12 \mathrm{~h}, 24 \mathrm{~h}$ ). To obtain images on the longer timescales and with different spacecraft attitudes, a special purpose programme was used to mosaic those from individual DPHs.

\subsection{Results of the search}

No evidence was found for prompt gamma-ray emission from any of the 24 classical novae listed in Table 3 . No points satisfied both the above trigger conditions (lightcurve plus image detection). The distribution of the complete ensemble of SNR measurements $(\sim 750000$ points, including all the single DPH light curves and all the energy bands) agrees very well with a random distribution with an average value of zero. The same is true for the rebinned light curves. We therefore conclude that in each case either the expected gamma-ray emission from the novae occurred when the object was not in the BAT FOV or the distance was too large for the predicted emission to be detected or the gamma-ray luminosity of the nova is weaker than expected.

To quantify the limiting distance below which a particular nova could be detected by BAT assuming the fluxes presented in Sect. 2, we first estimated a limiting flux for each point of the lightcurves for each nova. Lightcurves at different energies and timescales were considered separately in order to identify which combination of energy bin and timescale was most constraining.

Not only is the BAT coverage not continuous, but it also varies both in effective exposure and in the observing sequence from a source to another. As pointed out above, the peak of the expected gamma-ray flux of a nova could be missed during a coverage gap. Because the exact time of the peak is unknown, it is only possible to give a probability that emission at a particular level would have been detected, assuming that the peak occurred at a random, unknown, time. What can be done is to say that for 

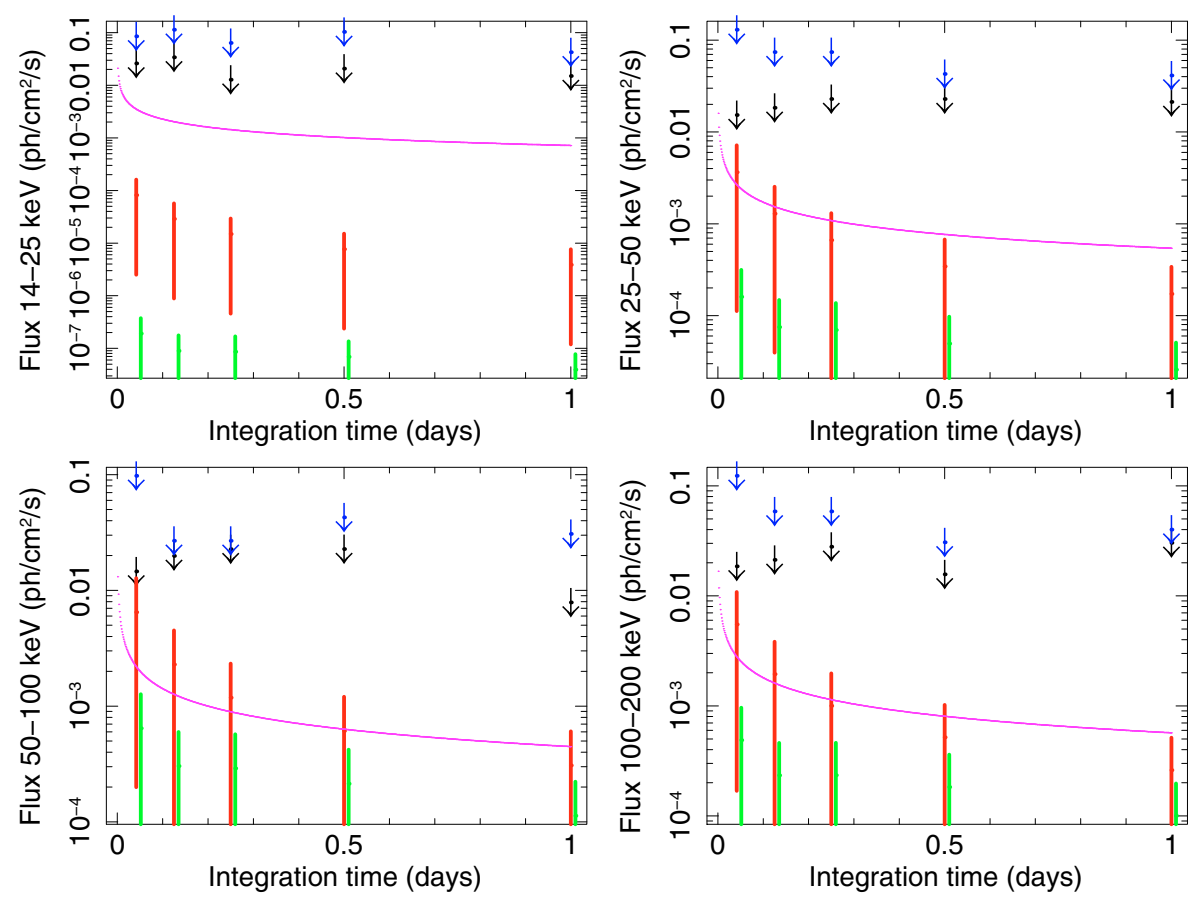

Fig. 6. Average expected flux as a function of integration time for the nova V2362 Cyg. Red and green bars are supposing a $\mathrm{CO}$ type and a ONe type nova respectively. Upper limits from BAT are indicated by arrows, for percentage coverage of possible onset times $P=10 \%$ (black) and $P=50 \%$ (blue). The purple curve represents the ideal $5 \sigma$ BAT sensitivity if the source were observed continuously in the fully coded FOV. As expected, for long integrations the sensitivity is better, but the average flux lower.

a certain percentage, $P$, of possible onset times, then the sensitivity and the coverage are such that the nova would have been detected had it been emitting at the level discussed in Sect. 2 and had it been closer than a distance $d$. Where a distance estimate is available it can then be compared with $d$. The calculation was done for $P=10 \%$ and for $P=50 \%$.

We illustrate this process in the case of nova V2362 Cyg in Fig. 6. BAT upper limits are indicated with an arrow. Black symbols show the limits which can be placed on the flux for a percentage $P=10 \%$ of the time, blue ones those for $P=50 \%$. The red lines (CO type) and green lines (ONe type) show the expected flux for a $\mathrm{CO}$ nova at the minimum $(1.5 \mathrm{kpc})$ and maximum $(12 \mathrm{kpc}$ ) of the various published distance estimates (see Table 3). For each binsize, these fluxes were obtained by averaging the appropriate curve in Fig. 1 from the beginning of the outburst up to a time equal to the binsize. The purple curves represent the best possible $5 \sigma$ BAT sensitivity, calculated from Eq. (1) using a typical value of $r_{\mathrm{B}}(E)$, if the source were seen continously in the fully coded FOV.

It can be seen that under ideal conditions the nova should have been detectable in the higher energy bands if its distance was toward the lower end of the range of estimates. However the actual upper limits are about an order of magnitude higher than the ideal ones because the nova was in the BAT FOV only for a fraction of the time and then in regions towards the edge having reduced sensitivity. Thus the non-detection is not surprising.

Table 4 gives, for each of the 24 novae examined, the best constraining timescale $\left(\Delta t_{\text {best }}\right)$, the measured $5 \sigma$ upper limits on the flux, and an estimate of the maximum distance out to which a CO nova should have been detectable $\left(d_{\max }\right)$. The limits are for the 50-100 keV band, which was the best constraining one in each case. When the nova type is unknown, the limiting distance for a ONe nova is indicated into parentheses. The limits and distances are given for two cases - those that can be placed for at least $10 \%$ or for at least $50 \%$ of the possible times of explosion. In no case was the non-detections surprising, though for several a detection might have been marginally possible in the most favourable conditions - if the distance was at the lower end of the range of estimates and the time of explosion were luckily placed with respect to the observations.

\subsection{Recurrent and dwarf novae}

In parallel with the searches for gamma-ray emission from classical novae, the same technique was applied to certain other objects. This lead to the detection of hard X-ray emission from both the recurrent nova RS Oph (reported in Bode et al. 2006) and the dwarf nova V455 And (Senziani et al. 2008). The strength and timescale of the high energy outburst from each of these objects were such that neither was detected on-board or by the routine monitoring on the ground (though more sophisticated analysis ground analysis now being developed might have done so). However a directed search, knowing the exact location and the approximate time of the event, allowed convincing detections.

In each case the strongest emission was below $25 \mathrm{keV}$ with little or no flux at higher energies. This is the inverse of the situation expected for classical novae and the physical processes involved are clearly different. In the case of RS Oph the emission was due to the shock of the ejecta with the surrounding medium, whereas the emission seen from V455 And was probably due to an instability in the accretion disc. However these detections demonstrate the potential of retrospective searches such as those described here.

\section{Discussion}

In Sect. 4.2 it was concluded that the BAT should be capable of detecting the gamma-ray emission due to nucleosynthesis in 2-5 novae during a $10 \mathrm{yr}$ life. This estimate depends on a number of assumptions. The most basic of these is the predicted gamma-ray flux during nova explosions, which mainly depends on the expected nucleosynthesis and the hydrodynamics of the expanding envelope. Indeed the possibility of confirming our current understanding of nucleosynthesis and dynamical evolution taking place in nova explosions is one of the incentives of this work. 
Table 4. Summary of the results obtained from BAT data for 24 Classical novae.

\begin{tabular}{|c|c|c|c|c|c|c|c|c|c|c|c|}
\hline \multirow[b]{2}{*}{ nova } & \multirow[b]{2}{*}{ year } & \multirow[b]{2}{*}{$\begin{array}{l}\quad m_{V} \\
\text { at visual max }\end{array}$} & \multirow[b]{2}{*}{$\begin{array}{c}t_{2} \\
\text { (days) }\end{array}$} & \multirow[b]{2}{*}{$E(B-V)$} & \multirow[b]{2}{*}{$\begin{array}{c}d_{\text {MMRD }} \\
(\mathrm{kpc})\end{array}$} & \multicolumn{3}{|c|}{ 10\% coverage time } & \multicolumn{3}{|c|}{$50 \%$ coverage time } \\
\hline & & & & & & $\Delta t_{\text {best }}$ & 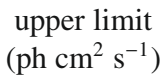 & $\begin{array}{l}d_{\max } \\
(\mathrm{kpc})\end{array}$ & $\Delta t_{\text {best }}$ & 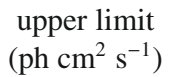 & $\begin{array}{l}d_{\max } \\
(\mathrm{kpc})\end{array}$ \\
\hline V2361 Cyg & 2005 & 10 & $5.5 \pm 0.5$ & 1.2 & $10.80_{-2.30}^{+2.91}$ & $\mathrm{DPH}$ & $5.9 \times 10^{-3}$ & 3.8 & $\mathrm{DPH}$ & $1.5 \times 10^{-2}$ & 2.3 \\
\hline V382 Nor & 2005 & 9.7 & $14.5 \pm 2.5$ & $1.50-1.51(*)$ & $4.8_{-1.2}^{+1.80}$ & $\mathrm{DPH}$ & $2.1 \times 10^{-2}$ & $1.8(0.5)$ & $\mathrm{DPH}$ & $2.6 \times 10^{-2}$ & $0.1(0.1)$ \\
\hline V378 Ser & 2005 & 11.6 & $52 \pm 18$ & 0.74 & $18.88_{-450}^{-1.2}$ & $\mathrm{DPH}$ & $1.1 \times 10^{-2}$ & 2.7 & $\mathrm{DPH}$ & $4.7 \times 10^{-2}$ & 0.1 \\
\hline V5115 Sgr & 2005 & 7.75 & $4 \pm 2$ & 0.53 & $10.22_{-2.38}^{+3.50}$ & $\mathrm{DPH}$ & $6.0 \times 10^{-3}$ & $3.7(0.9)$ & $\mathrm{DPH}$ & $2.4 \times 10^{-2}$ & $0.2(0.2)$ \\
\hline V1663 Aql & 2005 & 10.7 & $14 \pm 6$ & 2 & $3.88_{-1.38}^{+1.38}$ & $\mathrm{DPH}$ & $4.5 \times 10^{-3}$ & $4.1(1.0)$ & $12 \mathrm{~h}$ & $2.7 \times 10^{-3}$ & $0.1(0.2)$ \\
\hline V5116 Sgr & 2005 & 7.2 & $7 \pm 4$ & $0.34-0.57(*)$ & $8.3_{-3.1}^{+5.38}$ & $\mathrm{DPH}$ & $1.2 \times 10^{-2}$ & $0.1(0.1)$ & $\mathrm{DPH}$ & $1.2 \times 10^{-2}$ & $0.1(0.1)$ \\
\hline V1188 Sco & 2005 & 8.9 & $12 \pm 5$ & $1.09-1.49(*)$ & $4.4_{-1.9}^{+4.8}$ & $\mathrm{DPH}$ & $5.2 \times 10^{-3}$ & $3.9(1.0)$ & $\mathrm{DPH}$ & $5.6 \times 10^{-2}$ & $0.6(0.2)$ \\
\hline V1047 Cen & 2005 & 7.4 & $4.5 \pm 1.5$ & $1.28-1.38(*)$ & $2.8 \pm 0.5$ & DPH & $1.0 \times 10^{-2}$ & $2.7(0.7)$ & $\mathrm{DPH}$ & $2.6 \times 10^{-1}$ & $0.2(0.2)$ \\
\hline V476 Sct & 2005 & 11.4 & $12 \pm 2$ & 2.0 & $5.69_{-143}^{+1.85}$ & $1 \mathrm{~h}$ & $2.4 \times 10^{-2}$ & $1.8(0.5)$ & $\mathrm{DPH}$ & $2.4 \times 10^{-1}$ & $0.1(0.1)$ \\
\hline V477 Sct & 2005 & 10.75 & $7.5 \pm 2.5$ & 1.3 & $12.74_{-3.16}^{+4.04}$ & $1 \mathrm{~h}$ & $9.0 \times 10^{-3}$ & $2.8(0.7)$ & $1 \mathrm{~h}$ & $5.0 \times 10^{-2}$ & $0.3(0.2)$ \\
\hline V2575 Oph & 2006 & 11 & $31 \pm 2$ & 1.5 & $5.63_{-1.28}^{+1.169}$ & $\mathrm{DPH}$ & $7.8 \times 10^{-3}$ & 3.2 & $\mathrm{DPH}$ & $4.1 \times 10^{-2}$ & 1.2 \\
\hline V5117 Sgr & 2006 & 9.9 & $59 \pm 11$ & $0.5 \pm 0.15$ & $11.91_{-0.45}^{+0.63}$ & $1 \mathrm{~h}$ & $1.3 \times 10^{-2}$ & $2.5(0.6)$ & $1 \mathrm{~h}$ & $2.2 \times 10^{-1}$ & $0.3(0.2)$ \\
\hline V2362 Cyg & 2006 & 7.75 & $7 \pm 2.5$ & 0.59 & $8.90_{-2.19}^{+2.81}$ & $\mathrm{DPH}$ & $1.4 \times 10^{-2}$ & 2.3 & $1 \mathrm{~h}$ & $1.2 \times 10^{-1}$ & 0.6 \\
\hline V2576 Oph & 2006 & 9.2 & $25.5 \pm 2.5$ & 0.62 & $9.58_{-2.37}^{+3.39}$ & $\mathrm{DPH}$ & $8.9 \times 10^{-3}$ & 3.0 & $\mathrm{DPH}$ & $4.6 \times 10^{-2}$ & 1.3 \\
\hline V1065 Cen & 2007 & 8.7 & $19.5 \pm 1$ & $0.77-0.84(*)$ & $7.55_{-1.15}^{+1.65}$ & $1 \mathrm{~h}$ & $7.6 \times 10^{-3}$ & $3.2(0.8)$ & $1 \mathrm{~h}$ & $2.7 \times 10^{-2}$ & $1.6(0.4)$ \\
\hline V1280 Sco & 2007 & 4 & $13 \pm 1$ & $0.39-0.55(*)$ & $2.1 \pm 0.4$ & $1 \mathrm{~h}$ & $9.1 \times 10^{-3}$ & $3.0(0.8)$ & $\mathrm{DPH}$ & $2.7 \times 10^{-2}$ & $1.6(0.4)$ \\
\hline V1281 Sco & 2007 & 8.8 & $8 \pm 4$ & 0.7 & $12.10_{-3.37}^{+4.28}$ & $1 \mathrm{~h}$ & $9.1 \times 10^{-3}$ & $2.9(0.7)$ & $\mathrm{DPH}$ & $3.2 \times 10^{-2}$ & $1.7(0.4)$ \\
\hline V2467 Cyg & 2007 & 7.6 & $8 \pm 2$ & $1.6 \pm 0.1$ & $1.93_{-0.24}^{+0.36}$ & $1 \mathrm{~h}$ & $9.3 \times 10^{-3}$ & $2.9(0.7)$ & $\mathrm{DPH}$ & $5.3 \times 10^{-2}$ & $1.1(0.3)$ \\
\hline V2615 Oph & 2007 & 8.75 & $36.5 \pm 4.5$ & $1.0-1.3$ & $3.09_{-0.15}^{+0.24}$ & $1 \mathrm{~h}$ & $2.3 \times 10^{-2}$ & 1.9 & $\mathrm{DPH}$ & $9.3 \times 10^{-2}$ & 0.9 \\
\hline V5558 Sgr & 2007 & 6.5 & $6 \pm 1$ & 0.8 & $3.78_{-0.83}^{-0.06}$ & $1 \mathrm{~h}$ & $2.8 \times 10^{-2}$ & $1.7(0.5)$ & DPH & $1.1 \times 10^{-1}$ & $0.9(0.2)$ \\
\hline V598 Pup & 2007 & $?$ & $?$ & $?$ & $?^{-0.83}$ & $\mathrm{DPH}$ & $2.6 \times 10^{-2}$ & 1.8 & $\mathrm{DPH}$ & $1.1 \times 10^{-1}$ & 0.1 \\
\hline V390 Nor & 2007 & 9.8 & $49.5 \pm 5.5$ & 1.0 & $5.74_{-1.26}^{+1.67}$ & $1 \mathrm{~h}$ & $2.1 \times 10^{-2}$ & $1.9(0.5)$ & $1 \mathrm{~h}$ & $6.7 \times 10^{-2}$ & $1.0(0.3)$ \\
\hline V458 Vul & 2007 & 8.1 & $8.5 \pm 3.5$ & 0.6 & $10.01_{-2.71}^{+3.45}$ & $1 \mathrm{~h}$ & $2.8 \times 10^{-2}$ & $1.7(0.4)$ & $\mathrm{DPH}$ & $1.1 \times 10^{-1}$ & $0.4(0.1)$ \\
\hline V597 Pup & 2007 & $?$ & $?$ & 0.3 & $?^{-2 . / 1}$ & $1 \mathrm{~h}$ & $2.2 \times 10^{-2}$ & $1.8(0.5)$ & DPH & $9.8 \times 10^{-2}$ & $0.8(0.2)$ \\
\hline
\end{tabular}

As explained in Sect. 2, the expected gamma-ray flux from a nova depends critically on nuclear reactions rates which are still uncertain. Some alternative assumptions could lead to reduced expectations and better measurements of certain cross-sections are needed. The WD mass is another important parameter to consider. In this paper we obtained our predictions assuming that all CO novae and ONe novae hosted a $1.15 M_{\odot}$ or a $1.25 M_{\odot}$ WD respectively. In this scenario $\mathrm{CO}$ novae are more likely to be detected, but different fluxes could be obtained for different WD masses, as shown in Hernanz et al. (2002). WD masses, as well as the proportion between $\mathrm{CO}$ and ONe novae in the Galaxy, are very difficult to measure, and only rough estimations based on scarce information are available.

The results presented in Sect. 4.1 are based on the spatial distribution of novae in the Galaxy of model 1 of Table 1 . It is noteworthy that the conclusions do not strongly depend on the model chosen; those obtained with models 2 and 3 are very similar. This is because BAT could only detect novae within $\sim 5 \mathrm{kpc}$, where the spatial distributions are similar (see Fig. 3).

The other factor that can strongly affect our results is the rate of novae in the Galaxy, or more particularly the rate of discovered novae. It is certain that some relatively nearby novae are never observed, because they were too close to the sun, though chance of cloud coverage, or (particularly in the southern hemisphere) simply because no one was looking. The estimated discovery probability of $50 \%$ for novae $m_{V}<9$ given in Sect. 4.2 is based on $20 \mathrm{yr}$ of data. The rate of discovery and the methods used are evolving quickly and it is difficult to extract statistics on the distribution and characteristics of those currently being found. Almost certainly the chance that a nova potentially detectable with BAT will be reported is already higher.
The fact that no novae were detected in the first $3 \mathrm{yr}$ of BAT operations (Sect. 5) is consistent with the expectation of 2-5 nova in 10 years estimated in Sect. 4.2. None of the 24 novae occurring during that time was sufficiently close and sufficiently well covered that a detection was likely. The fact that the technique was able to detect similar outbursts from RS Oph and V455 And demonstrates its efficacy even though the emission had a different origin. The search is being continued and the analysis presented here gives high hopes of eventual success.

An intriguing possibility is that a sufficiently nearby nova might be detectable without depending on the later detection of an optical nova. If it were found by the on-board software, rather than later on the ground, it might even trigger a spacecraft slew to bring the position within the field of view of the XRT and UVOT instruments. Because the gamma rays are hardly affected by interstellar dust, it could even lead to the discovery of a nova that would otherwise be unobserved (IR observations would allow the confirmation of the nature of the event). The probability of a nova so close is, of course relatively low, but the information that could be obtained would be of enormous value.

Acknowledgements. The authors wish to thank members of the Swift BAT team for useful discussions and advice. We acknowledge with thanks the variable star observations from the AAVSO International Database contributed by observers worldwide and used in this research. This work was supported by the Centre National d'Etudes Spatiales (CNES). It is based on observations with BAT embarked on SWIFT. Financial support from MEC ESP2007-61593, AGAUR 2005-SGR00378 and FEDER funds is also acknowledged.

\section{References}

Barthelmy, S. D., Barbier, L. M., Cummings, J. R., et al. 2005, Space Sci. Rev., 120,143

Bode, M. F., O’Brien, T. J., Osborne, J. P., et al. 2006, ApJ, 652, 629 
Boyd, D., \& Poyner, G. 2006, Journal of the British Astronomical Association, 116,320

Chafa, A., Tatischeff, V., Aguer, P., et al. 2005, Phys. Rev. Lett., 95, 031101

Clayton, D. D., \& Hoyle, F. 1974, ApJ, 187, L101

Coc, A., Hernanz, M., José, J., \& Thibaud, J.-P. 2000, A\&A, 357, 561

Czart, K., Lewandowski, M., Maciejewski, G., et al. 2006, The Astronomer's Telegram, 792, 1

Darnley, M. J., Bode, M. F., Kerins, E., et al. 2006, MNRAS, 369, 257

Dawson, P. C., \& Johnson, R. G. 1994, JRASC, 88, 369

Della Valle, M., \& Livio, M. 1995, ApJ, 452, 704

Downes, R. A., Webbink, R. F., Shara, M. M., et al. 2001, PASP, 113, 764

Fenimore, E. E., Palmer, D., Galassi, M., et al. 2003, in Gamma-Ray Burst and Afterglow Astronomy 2001: A Workshop Celebrating the First Year of the HETE Mission, ed. G. R. Ricker, \& R. K. Vanderspek, American Institute of Phys. Conf. Ser., 662, 491

Gehrels, N., Chincarini, G., Giommi, P., et al. 2004, ApJ, 611, 1005

Gómez-Gomar, J., Hernanz, M., José, J., \& Isern, J. 1998, MNRAS, 296, 913

Hakkila, J., Myers, J. M., Stidham, B. J., \& Hartmann, D. H. 1997, AJ, 114, 2043

Harris, M. J., Naya, J. E., Teegarden, B. J., et al. 1999, ApJ, 522, 424

Harris, M. J., Teegarden, B. J., Cline, T. L., et al. 2000, ApJ, 542, 1057

Hatano, K., Branch, D., Fisher, A., \& Starrfield, S. 1997, MNRAS, 290, 113

Hernanz, M., \& José, J. 2004, in 5th INTEGRAL Workshop on the INTEGRAL

Universe, ed. V. Schoenfelder, G. Lichti, \& C. Winkler, ESA SP, 552, 95

Hernanz, M., \& José, J. 2006, New Astron. Rev., 50, 504

Hernanz, M., José, J., Coc, A., Gómez-Gomar, J., \& Isern, J. 1999, ApJ, 526, L97

Hernanz, M. J., Smith, D. M., Fishman, J., et al. 2000, in Amer. Inst. Phys. Conf. Ser., ed. M. L. McConnell, \& J. M. Ryan, 510, 82

Hernanz, M., Gómez-Gomar, J., \& José, J. 2002, New Astron. Rev., 46, 559

Jean, P., Hernanz, M., Gómez-Gomar, J., \& José, J. 2000, MNRAS, 319, 350

José, J., \& Hernanz, M. 1998, ApJ, 494, 680
Kent, S. M., Dame, T. M., \& Fazio, G. 1991, ApJ, 378, 131

King, I., Gilmore, G., \& van der Kruit, P. C. 1990, The Milky Way As Galaxy, ed. I. King, G. Gilmore, \& P. C. van der Kruit (University Science Books), 392

Lane, B. F., Retter, A., Eisner, J. A., Thompson, R. R., \& Muterspaugh, M. W. 2006, in Advances in Stellar Interferometry, ed. J. D. Monnier, M. Schöller, \& W. C. Danchi, Proc. SPIE, 6268

Livio, M., \& Truran, J. W. 1994, ApJ, 425, 797

Munari, U., Henden, A., Pojmanski, G., et al. 2006a, MNRAS, 369, 1755

Munari, U., Siviero, A., Navasardyan, H., \& Dallaporta, S. 2006b, A\&A, 452, 567

Osborne, J. P., Page, K., Starrfield, S., et al. 2007, The Astronomer's Telegram, 1011,1

Poggiani, R. 2006, Astron. Nachr., 327, 895

Read, A. M., Saxton, R. D., \& Esquej, P. 2007a, The Astronomer's Telegram, 1282,1

Read, A. M., Saxton, R. D., Osborne, J. P., et al. 2007b, The Astronomer's Telegram, 1301, 1

Senziani, F., Skinner, G., \& Jean, P. 2008, The Astronomer's Telegram, 1372, 1 Shafter, A. W. 1997, ApJ, 487, 226

Skinner, G. K. 2008, Appl. Opt., submitted

Smith, D. M. 2004, in 5th INTEGRAL Workshop on the INTEGRAL Universe, ed. V. Schoenfelder, G. Lichti, \& C. Winkler, ESA SP, 552, 45

Starrfield, S., Truran, J. W., \& Sparks, W. M. 1978, ApJ, 226, 186

Steeghs, D., Greimel, R., Drew, J., et al. 2006, The Astronomer's Telegram, 795, 1

Steeghs, D., Drew, J., Greimel, R., et al. 2007, The Astronomer's Telegram, 1031, 1

Swank, J. H. 2007, The Astronomer's Telegram, 1010, 1

Torres, M. A. P., Jonker, P. G., Challis, P., et al. 2007, The Astronomer's Telegram, 1285, 1 